Clowes, R. C. (1958). J. gen. Microbiol. 18, 140-153

\title{
Nutritional Studies of Cysteineless Mutants of Salmonella typhimurium
}

\author{
By R. C. CLOWES \\ Department of Genetics, Carnegie Institution of Washington, \\ Cold Spring Harbor, N.Y., U.S.A.*
}

\begin{abstract}
SUMMARY: Forty-three separately isolated cyst(e)ineless mutants of Salmonella typhimurium were investigated biochemically. Six main phenotypes were identified, accommodating thirty-nine of the mutants; the remaining four mutants were of intermediate phenotype. The phenotypes are suggested to result from a series of five metabolic blocks in a pathway of cyst(e)ine synthesis in which sulphate can be reduced by two alternative routes, in both of which most of the intermediates are inorganic.
\end{abstract}

In this paper the expression cyst(e)ine is used to represent cysteine or cystine. The pathway of cyst(e)ine biosynthesis has previously been studied in a variety of micro-organisms including Escherichia coli (Lampen, Roepke \& Jones, 1947; Roberts et al. 1955; Simonds 1948); Neurospora crassa (Phinney, 1948; Horowitz, 1950, 1955); Aspergillus nidulans (Hockenhull, 1949; Shepherd, 1956); $A$. niger (Steinberg, 1941). In most of these studies, the data are consistent with an inorganic pathway of sulphate reduction through sulphite and thiosulphate, and only after passing through these inorganic intermediates is the reduced sulphur atom combined with a carbon chain to form cyst(e)ine. The carbon chain has been suggested by Roberts et al. (1955) to be provided by serine, which was also indicated by the data of Hockenhull (1949). The irreversibility of the cyst(e)ine-methionine pathway in Escherichia coli was apparent from the data of both Simmonds (1948) and Roberts et al. (1955), although methionine was able to replace cysteine in the $\boldsymbol{E}$. coli mutants studied by Lampen et al. (1947), and also in Aspergillus and Neurospora. In all cases, other inorganic sources of sulphur, wherever studied, gave analogous growth responses to either sulphate, sulphite or thiosulphate, and were thus considered to be rapidly converted to one of these compounds and not to be direct intermediates. Moreover, thiosulphate satisfied the growth of almost all mutants able to utilize cyst(e)ine. The data of Hockenhull (1949) and Horowitz (1955) showed that cysteic acid could replace sulphite. However, cysteine sulphinic acid could not replace thiosulphate, as would be expected if this were a direct intermediate, but had the same growth-promoting activity as the more oxidized cysteic acid. It was therefore suggested by these authors that the initial steps of the pathway involved only inorganic ions until the sulphur had been reduced to the state of thiosulphate. However, Shepherd (1956) isolated certain mutants of Aspergillus nidulans which were incapable of growth with

* Present address: M.R.C. Microbial Genetics Research Unit, Hammersmith Hospital, Ducane Road, London, W. 12. 
sulphite and in some instances with thiosulphate, but which were able to utilize either cysteic acid or cysteine sulphinic acid. He therefore postulated alternative organic and inorganic pathways of sulphur reduction in which either serine, pyruvate or cysteic acid could act as alternative carbon sources.

This paper describes the differentiation of a series of cyst(e)ineless mutants of Salmonella typhimurium by biochemical tests, from which some indication of the mode of cyst(e)ine biosynthesis by this organism may be obtained.

\section{METHODS}

\section{Media}

Defined medium. The minimal medium of Davis (Lederberg, 1950) was used, supplemented as indicated with various sulphur sources, at a concentration of $10 \mu \mathrm{g}$. S $/ \mathrm{ml}$. (unless otherwise specified), or with amino acids $(20 \mu \mathrm{g} . / \mathrm{ml}$.). It contained $240 \mu \mathrm{g}$. S/ml. as sulphate.

Nutrient agar (Difco) was used when a complete medium was required.

Sulphur-free ( $S$-free) medium, used when a medium deficient in all sulphur sources was required, was made up as tris (2-amino-2-hydroxymethylpropane1:3 diol) glucose medium (Hershey, 1955), with the omission of sulphate.

Enriched minimal agar, used in syntrophism experiments, consisted of minimal agar containing $0.01 \%$ nutrient broth powder (Demerec \& Cahn, 1953).

\section{Organisms}

The cyst(e)ineless mutants used in this study were auxotrophs derived from Salmonella typhimurium, strains LT-2 and LT-7 (Zinder \& Lederberg, 1952), isolated by various members of the Department of Genetics, Carnegie Institution of Washington, and made available by Dr M. Demerec.

The wild-type strains grew well on the minimal medium in which sulphate was the sole sulphur source. A series of auxotrophic mutants were isolated from both u.v.-irradiated and non-irradiated cultures of the wild-type strains by penicillin screening (Lederberg, 1950). From the auxotrophs, detected by their ability to grow on nutrient agar but not minimal agar, the cyst(e)ineless mutants were identified by their growth when L-cysteine or L-cystine was added to the minimal medium. They were designated as described by Demerec (1956) by the prefix cys, followed by a figure denoting the sequence of isolation; e.g.cys-1. In some instances, previously isolated auxotrophic strains including tryptophanless $(t r y)$ and prolineless ( $p r o$ ) mutants were used as parental strains for the isolation of double auxotrophs. The derivation of the mutants are listed in Table 1.

Nutritional requirements of mutant strains were established auxanographically; $0 \cdot 1 \mathrm{ml}$. samples of an overnight broth culture diluted $10^{-3}$ were spread over the surface of minimal agar plates. The agar was then removed at several places by a sterile cork-borer and drops of sterile solutions of the supplements placed into the holes. After incubation for $40 \mathrm{hr}$. at $37^{\circ}$, the presence or absence of haloes of growth around the holes was noted. Alternatively, the strains were spread, after appropriate dilutions, on minimal agar plates 
supplemented with the growth factor. After incubation at $37^{\circ}$ for $18 \mathrm{hr}$., the plates were examined for the presence or absence of colonies of Salmonella typhimurium.

Table 1. Sources of stocks of cyst(e)ineless mutants of Salmonella typhimurium

\begin{tabular}{|c|c|c|c|}
\hline \multicolumn{2}{|c|}{ U.v.-irradiated* } & \multicolumn{2}{|c|}{ Non-irradiated } \\
\hline Source & Mutants & Source & Mutants \\
\hline LT-2 (wild-type) & $\begin{array}{l}\text { cys-1, }-2,-3,-4,-5,-6,-7 \\
-8,-10,-11,-12,-13,-14 \\
-15,-16,-17,-18,-20 \\
-21,-22,-23,-24\end{array}$ & $\begin{array}{l}\text { LT-2 (wild-type) } \\
\text { LT-2 try-8 }\end{array}$ & $\begin{array}{l}\text { cys }-36,-37,-38 \\
\text { cys }-40,-41,-42 \\
-43,-44,-50,-51\end{array}$ \\
\hline $\begin{array}{l}\text { LT-2 pro- } 6 \\
\text { LT-7 (wild-type) }\end{array}$ & $\begin{array}{l}\text { cys- } 25,-27,-28,-29 \\
\text { cys }-30,-32\end{array}$ & $\begin{array}{l}\text { LT-2 try-2 } \\
\text { LT-2 try-7 }\end{array}$ & $\begin{array}{l}\text { cys }-45,-46 \\
\text { cys }-47,-48,-49\end{array}$ \\
\hline
\end{tabular}

* The designation 'U.v.-irradiated' indicates that the mutants listed were obtained from cultures which had been exposed to u.v. radiation. However, since auxotrophic mutants from such cultures were isolated with no greater frequency than from non-irradiated cultures, it is likely that the mutants from both sources are largely 'spontaneous' in origin.

Syntrophism between strains was tested in two ways:

(a) By the parallel-streak method between pairs of mutants. Overnight broth cultures of the two mutants were diluted and streaks were made in parallel about $0.5 \mathrm{~cm}$. apart on enriched minimal agar. After incubation for 5 days at $37^{\circ}$, the growth was then compared with single-streak controls.

(b) By auxanograph testing of culture filtrates and disrupted organisms. Overnight broth cultures were washed, re-inoculated into liquid minimal medium containing $4 \mu \mathrm{g}$. L-cysteine $/ \mathrm{ml}$. to give about $5 \times 10^{8}$ bacteria $/ \mathrm{ml}$., and aerated for $18 \mathrm{hr}$. at $37^{\circ}$. By this method the required amino acid (cyst(e)ine) was exhausted from medium and organisms; no significant reversion to prototrophy occurred at this high density of inoculation. The organisms were then centrifuged down, washed, concentrated to $2 \times 10^{10} / \mathrm{ml}$. in minimal medium, and $10 \mathrm{ml}$. samples disrupted for $2 \mathrm{~min}$. in a 'Raytheon' sonic oscillator, after which time the viable count had fallen to $10^{7} / \mathrm{ml}$. The supernatant fluids from the cultures in limiting cysteine-supplemented medium and the complete disruption products were then tested auxanographically for their ability to support the growth of other mutants.

Groweth curves were plotted from cultures aerated at $37^{\circ}$ in S-free media with various supplements, inoculated with about $2 \times 10^{8}$ washed organisms $/ \mathrm{ml}$. Growth was measured as optical density by means of a Klett-Summerson photoelectric colorimeter which was calibrated with a culture of the wild-type strain LT-2 in the late logarithmic phase of growth. Optical densities of serial twofold dilutions of this culture were plotted against the corresponding numbers of viable organisms $/ \mathrm{ml}$. obtained by counts of colonies appearing on plates of nutrient agar spread with appropriate dilutions of the culture and incubated at $37^{\circ}$ for $18 \mathrm{hr}$. The optical density varied linearly with the number of organisms $/ \mathrm{ml}$. between the values of $5 \times 10^{7}$ and $2 \times 10^{9}$, organisms/ml. 
Isotopic competition studies used carrier-free ${ }^{35} \mathrm{~S}$-sulphate (Oak Ridge National Laboratory; kindly supplied by Dr A. D. Hershey).

\section{RESULTS}

\section{Growth of mutant strains on solid media}

The cyst(e)ineless mutants were first tested for ability to utilize sulphate, sulphite, thiosulphate or cysteine. All strains were unable to utilize sulphate but responded to added cysteine. However, when broth cultures of certain mutants were plated on minimal agar $\left(c .10^{8}\right.$ organisms/plate $)$ in which sulphate was the sole sulphur source, at least several dozen prototrophic colonies were

\section{Table 2. Growth of cyst(e)ineless mutants of Salmonella typhimurium in the presence of various sulphur sources}

All sulphur sources provided to give $10 \mu \mathrm{g}$. S/ml. (except 'thiosulphate (a)' provided at $1 \mathrm{mg}$. S/ml.) incorporated into minimal agar. Plates were incubated for $18 \mathrm{hr}$. at $37^{\circ}$ after inoculation.

Sulphur source

Cysteine

Sulphate $\left(\mathrm{SO}_{4}{ }^{\prime \prime}\right)$

Sulphite $\left(\mathrm{SO}_{3}{ }^{\prime \prime}\right)$

Thiosulphate $\left(\mathrm{S}_{\mathbf{2}} \mathrm{O}_{\mathbf{3}}{ }^{\prime \prime}\right)$

Thiosulphate $\left(\mathrm{S}_{2} \mathrm{O}_{3}{ }^{\prime \prime}\right)(a)$

Lanthionine

Cysteic acid

Dithionate $\left(\mathrm{S}_{2} \mathrm{O}_{6}{ }^{\prime \prime}\right)$

Cysteine sulphinic acid

Metabisulphite $\left(\mathrm{S}_{2} \mathrm{O}_{8}{ }^{\prime \prime}\right)$

Sulphur (S)

Sulphide $\left(\mathbf{S}^{\prime \prime}\right)$

Dithionite $\left(\mathrm{S}_{2} \mathrm{O}_{4}{ }^{\prime \prime}\right)$

$\overbrace{\text { cys } A \quad c y s B \quad \begin{array}{c}\text { cysC } C \quad c y s D \\ \text { Growth response }\end{array}}^{\text {Phenotype }}$

\begin{tabular}{ccccc}
\hline++ & +++ & +++ & +++ & +++ \\
- & - & - & - & - \\
++ & - & +++ & - & - \\
- & - & +++ & +++ & - \\
++ & +++ & +++ & +++ & - \\
+++ & +++ & +++ & +++ & +++ \\
- & - & - & - & - \\
- & - & - & - & - \\
++ & - & +++ & - & - \\
++ & - & +++ & - & - \\
+ & - & + & + & - \\
+ & - & + & + & - \\
++ & - & +++ & ++ & -
\end{tabular}

$+++=$ growth equivalent to that with cysteine supplement; $++=$ growth slightly less than that with cysteine supplement; $+=$ growth greatly decreased compared to that with cysteine supplement; $-=$ no apparent growth.

produced. This high frequency of prototroph production made nutritional studies difficult and no further investigations were made with these strains (cys-9, $-19,-26,-31,-35$ and -39). The growth responses shown in Table 2 (lines 3 and 4) indicate that they can be separated into four classes exhibiting growth on:

(a) sulphite only, cys-1, -3, -5, -13, -20, -21, -22 and -32 (designated cys $A$ mutants);

(b) on neither salt, cys-2, -4, -6, -8, -10, -11, -12, -14, -15, -16, -17, -18, -24, $-25,-27,-30,-40,-41$ and -45 (designated cys $B$ or $c y s E$ mutants);

(c) sulphite or thiosulphate, cys-7, $-38,-43$ and -47 (designated cysC mutants); or

(d) thiosulphate only, cys-23, $-28,-29,-36,-37,-42,-44,-46,-48,-49,-50$ and -51 (designated $c y s D$ mutants). 
In subsequent experiments the mutants were streaked on minimal agar + different concentrations of sulphite or thiosulphate at 20,50, 100, 200,500 and $1000 \mu \mathrm{g} . \mathrm{S} / \mathrm{ml}$. The response to all concentrations of sulphite up to $500 \mu \mathrm{g} . / \mathrm{ml}$. was the same as that at $10 \mu \mathrm{g} . / \mathrm{ml}$, concentrations above $500 \mu \mathrm{g} . / \mathrm{ml}$. being inhibitory. On thiosulphate supplemented media, the response at all concentrations was identical to that already reported at $10 \mu \mathrm{g} . / \mathrm{ml}$., except at the $1 \mathrm{mg} . / \mathrm{ml}$. level, at which concentration in addition to those mutants growing at lower concentrations, a certain number of other strains unable to utilise these lower concentrations were able to grow. These mutants, which respond to thiosulphate only at a concentration of $1 \mathrm{mg} . / \mathrm{ml}$., are designated cys $B$ mutants (cys-4, -10,-12, -14, -15, -16, -18, -24, -25, -27, -40,-41 and -45) and are differentiated by this criterion from those mutants which are unable to utilize thiosulphate at any concentration tested and which form the group designated cysE (cys-2, -6, -8, -11, -17 and -30). The mutants are thus differentiated into the five phenotypes cys $A, B, C, D$ and $E$, as shown in Table 2 by the growth responses on sulphite and thiosulphate. The growth responses were identical when the supplements were added to minimal medium (containing sulphate) or to S-free medium and, with certain exceptions discussed later, prolonged incubation up to $\mathbf{5}$ days caused no detectable change.

The ability of other sulphur sources to support growth was then investigated. Lanthionine ( $\beta$ - $\beta^{\prime}$-thiodialanine) allowed the growth of all mutants, but neither cysteic acid nor dithionate $\left(\mathrm{S}_{2} \mathrm{O}_{6}{ }^{\prime \prime}\right)$ stimulated the growth of any mutant. Both cysteine sulphinic acid and metabisulphite $\left(\mathrm{S}_{2} \mathrm{O}_{5}{ }^{\prime \prime}\right)$ supported the growth of the same mutants as did sulphite (cys $A$ and $c y s C)$. Elementary sulphur and sulphide $\left(\mathrm{S}^{\prime \prime}\right)$ gave no stimulation of some groups, and a slow and suboptimal growth with all other strains. Dithionite $\left(\mathrm{S}_{\mathbf{2}} \mathrm{O}_{\mathbf{4}}{ }^{\prime \prime}\right)$, however, was a satisfactory source of sulphur for all $c y s A, c y s C$ and $c y s D$ mutants.

The examination for syntrophism by the parallel-streaking method gave positive results only after 3 days of incubation at $37^{\circ}$, the plates were incubated for 5 days before scoring, when it was seen that certain $c y s D$ mutants (designated $D_{1}$ mutants and comprising cys-23, $-44,-46,-48,-50$ and -51 ) supported the growth of all mutants in the $c y s A$ and $c y s C$ groups. Other $c y s D$ mutants $\left(D_{2}\right.$ mutants, cys-28, $-29,-36,-37,-42$ and -49$)$ did not feed any other mutant strain.

Auxanograph testing of disrupted cell suspensions showed that disrupted cys $A$ cells fed $c y s C$ mutants, and that disrupted $c y s D_{1}$ cells fed either $c y s C$ or cys $A$ mutants. Furthermore, disrupted cells of $c y s C, c y s A, c y s B$ and $c y s E$ mutants fed $c y s D_{1}$ mutants. However, this latter stimulation of $c y s D_{1}$ mutants was also given both by disrupted wild-type cells and by the culture filtrates of both wild-type and of these mutants. Apart from this apparent non-specific stimulation of $c y s D_{1}$ mutants, the supernatant fluids had no growth-promoting activity on any mutant.

\section{Growth of wild-type Salmonella typhimurium in liquid culture}

Overnight broth cultures of wild-type strains LT-2 and LT-7 were washed in S-free medium and inoculated into similar medium, either unsupplemented or 
supplemented with a sulphur source at $10 \mu \mathrm{g}$. S/ml. (when stereoisomeric compounds were used, at $20 \mu \mathrm{g}$. S $/ \mathrm{ml}$.). The cultures were incubated with aeration at $37^{\circ}$, and the optical densities recorded (Fig. 1). The growth rates in cysteine, sulphate, sulphite or thiosulphate media were the same (mean generation time $c .50 \mathrm{~min}$.), attaining a maximum of $3 \times 10^{9} \mathrm{organisms} / \mathrm{ml}$. which was determined by the glucose content of the medium $(2 \mathrm{mg} . / \mathrm{ml}$.). The corresponding curves obtained with dithionite, DL-lanthionine or cysteine sulphinic acid

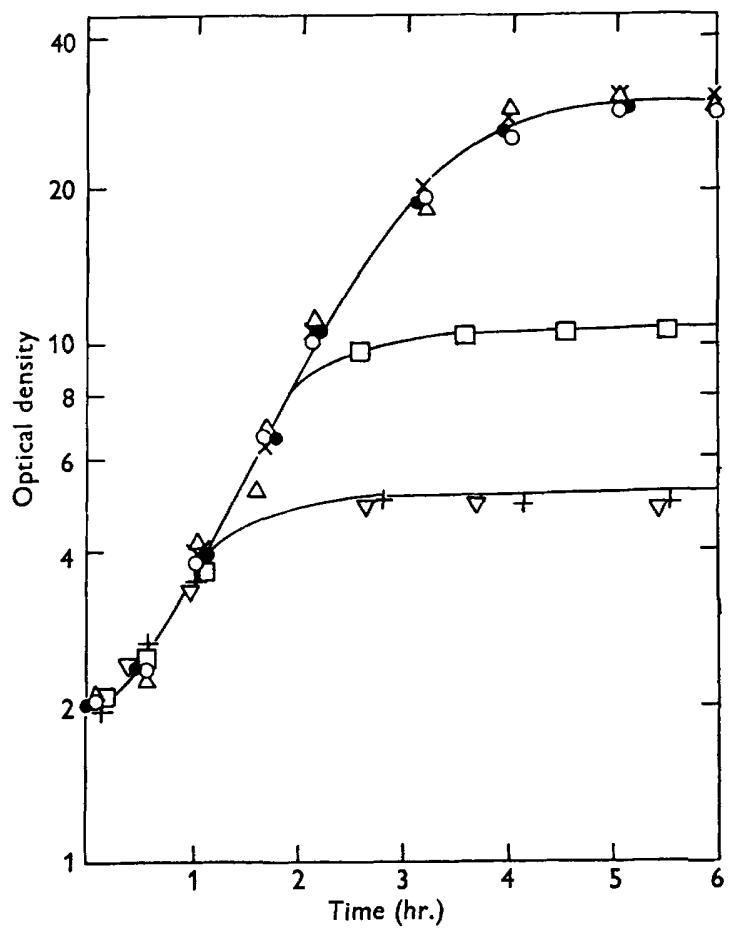

Fig. 1. Growth curves of wild-type Salmonella typhimurium, aerated at $37^{\circ}$ in liquid medium with cysteine, sulphate $\left(\mathrm{SO}_{4}{ }^{\prime \prime}\right)$, sulphite $\left(\mathrm{SO}_{3}{ }^{\prime \prime}\right)$, thiosulphate $\left(\mathrm{S}_{2} \mathrm{O}_{3}{ }^{\prime \prime}\right)$, methionine or cysteic acid as a sole sulphur source at equiv. $10 \mu \mathrm{g}$. S/ml. medium. Each unit of optical density corresponds to about $10^{8}$ bacteria/ml. O, Cysteine; $\times, \mathrm{SO}_{4}{ }^{\prime \prime} ; \Delta, \mathrm{SO}_{3}{ }^{\prime \prime}$; , $\mathrm{S}_{2} \mathrm{O}_{3}{ }^{\prime \prime} ;+$, S-free; $\square$, methionine; $\nabla$, cysteic acid.

(not shown in Fig. 1) were similar. In S-free medium, the organisms grew initially at the optimal growth rate until a population corresponding approximately to a twofold to threefold increase had been obtained. There was little further growth. Analogous results have been described with Escherichia coli by Roberts et al. (1955) who showed that growth in S-free medium was associated with a utilization of the internal sulphur reserves of the organisms, mainly in the form of glutathione.

When L-methionine was the sole sulphur source, the organisms grew for a period about twice that obtained in S-free medium, after which time the growth virtually ceased. This suggests that methionine cannot act as a sole sulphur source for Salmonellas, the increase in growth over that observed in 
S-free medium being due only to sparing of the internal sulphur reserves. Roberts et al. (1955) showed a similar phenomenon in Escherichia coli, and demonstrated that during growth $50 \%$ of the sulphur uptake was converted into methionine-containing protein, which would be consistent with these observations. When cysteic acid was the sole sulphur source, there was no growth beyond that obtained in S-free medium.

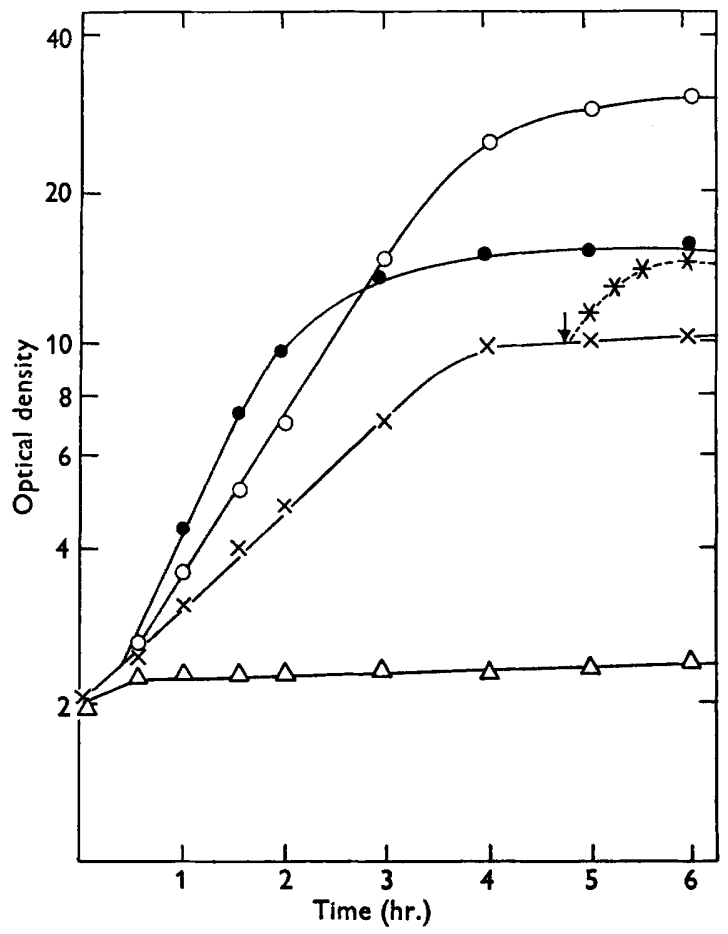

Fig. 2. Growth curves of cyst(e)ineless mutants of Salmonella typhimurium aerated at $37^{\circ}$ in liquid medium with cysteine, sulphate $\left(\mathrm{SO}_{4}{ }^{\prime \prime}\right)$, sulphite $\left(\mathrm{SO}_{3}{ }^{\prime \prime}\right)$, thiosulphate $\left(\mathrm{S}_{2} \mathrm{O}_{3}{ }^{\prime \prime}\right)$, cysteine sulphinic acid (CSA), cysteic acid (CA), or dithionite $\left(\mathrm{S}_{2} \mathrm{O}_{4}{ }^{\prime \prime}\right)$ as a sole source of sulphur. - $-\mathrm{O}-$ represents growth with cysteine (cys $A, B, C, D, E) ; \mathrm{S}_{2} \mathrm{O}_{3}^{\prime \prime}$ (cysC, cysD); $\mathrm{SO}_{3}{ }^{\prime \prime}, \mathrm{S}_{2} \mathrm{O}_{4}^{\prime \prime}\left(\right.$ cysC). $-\times-\times$ - represents growth with $\mathrm{SO}_{3}^{\prime \prime}($ cys A); or with $\mathrm{S}_{2} \mathrm{O}_{4}{ }^{\prime \prime}$ (cys $A$ and cysD). $-\triangle-\triangle-$ represents growth in either $\mathrm{S}$-free medium or with $\mathrm{SO}_{4}{ }^{\prime \prime}($ cys $A, B, C, D, E) ; \mathrm{SO}_{3}{ }^{\prime \prime}$ or $\mathrm{CSA}(c y s B, D, E) ; \mathrm{S}_{2} \mathrm{O}_{3}{ }^{\prime \prime}(c y s A, B, E)$; and $\mathrm{S}_{2} \mathrm{O}_{4}{ }^{\prime \prime}(c y s B$, $E)$, and - - represents growth of all mutants in broth. All sulphur sources provided at $10 \mu \mathrm{g} . \mathrm{S} / \mathrm{ml}$. The interrupted line shows the effect of addition at time marked by arrow of a further $10 \mu \mathrm{g}$. S/ml. of either $\mathrm{SO}_{3}{ }^{\prime \prime}$ or $\mathrm{S}_{2} \mathrm{O}_{4}{ }^{\prime \prime}$ to the medium. Each unit of optical density corresponds to $c .10^{8}$ bacteria $/ \mathrm{ml}$.

\section{Growth of mutant strains in liquid culture}

The growth of the cyst(e)ineless mutants in cysteine-supplemented liquid media was, in general, rather less rapid than that of the wild-type strain (Fig. 2), giving a mean generation time of about $65 \mathrm{~min}$., eventually attaining the same maximum of $3 \times 10^{9} \mathrm{organisms} / \mathrm{ml}$. In S-free medium or in sulphate medium no mutant of any group was able to grow to any significant extent. The growth of cysC strains in media supplemented with sulphite, thiosulphate, dithionite 
or cysteine sulphinic acid and that of $c y s D$ strains in thiosulphate-supplemented medium showed the same type of growth response as with the cysteine supplement. However, cys $A$ strains in sulphite or cysteine sulphinic acid-supplemented media, and $c y s A$ and $c y s D$ strains in dithionite-supplemented medium, grew more slowly (mean generation time about $100 \mathrm{~min}$.) and attained a lower maximum of $c .10^{9} \mathrm{organisms} / \mathrm{ml}$. No growth of $c y s A$ strains was observed in thiosulphate-supplemented medium, nor of $c y s D$ strains in sulphite or cysteine sulphinic acid-supplemented media. $C y s B$ and $c y s E$ strains could not grow on any of these sulphur sources except cysteine, in which case the growth response was similar to that given by other mutants with this sulphur source. All the mutant strains at first grew more rapidly in broth (mean generation time 35-40 min.) than in cysteine-supplemented defined medium, but there was little growth beyond $10^{9}$ organisms $/ \mathrm{ml}$. Further growth to the normal $\left(3 \times 10^{9}\right)$ yield could be achieved by the addition of cysteine $(5 \mu \mathrm{g} . / \mathrm{ml}$.$) . Since$ this apparent deficiency of cyst(e)ine in broth would tend to select any reversions to wild type, cyst(e)ineless mutants were grown in broth fortified with cysteine $(20 \mu \mathrm{g} . / \mathrm{ml}$.).

\section{Isotopic competition experiments}

Wild-type organisms, after overnight growth in broth at $37^{\circ}$ with aeration, were washed twice and inoculated into $\mathrm{S}$-free medium $+{ }^{35} \mathrm{~S}$-sulphate at $0.05 \mu \mathrm{c} / \mathrm{ml} .+{ }^{32} \mathrm{~S}$-sulphate at $3 \mu \mathrm{g}$. S $/ \mathrm{ml}$. to give $10^{8}$ organisms $/ \mathrm{ml}$. Methionine, cysteine, sulphite, dithionite, cysteic acid or thiosulphate (all at $3 \mu \mathrm{g} . \mathrm{S} / \mathrm{ml}$.) were added individually to parallel cultures. All cultures were incubated at

Table 3. Suppression of ${ }^{35}$ S-sulphate uptake by Salmonella typhimurium-LT2 in the presence of non-radioactive competitors

\begin{tabular}{|c|c|c|}
\hline \multirow{2}{*}{$\begin{array}{l}\text { Non-radioactive } \\
\text { supplement }(a)\end{array}$} & \multicolumn{2}{|c|}{$\begin{array}{c}\text { Uptake of radiosulphate } \\
\text { (\% of control) }\end{array}$} \\
\hline & (b) & (c) \\
\hline None (control) & 100 & 100 \\
\hline L-Cysteine & 1 & 1 \\
\hline Thiosulphate $\left(\mathrm{S}_{2} \mathrm{O}_{3}{ }^{\prime \prime}\right)$ & 2 & $\mathbf{3}$ \\
\hline Dithionite $\left(\mathrm{S}_{2} \mathrm{O}_{4}{ }^{\prime \prime}\right)$ & 17 & 60 \\
\hline Sulphite $\left(\mathrm{SO}_{3}{ }^{\prime \prime}\right)$ & 37 & - \\
\hline DL-Methionine & 40 & - \\
\hline Cysteic acid & 100 & - \\
\hline
\end{tabular}

(a) Sulphur concentration of non-radioactive compound, $3 \mu \mathrm{g} . / \mathrm{ml}$. medium.

(b) Total sulphate in each culture, $3 \mu \mathrm{g} . / \mathrm{ml}$. medium.

(c) Total sulphate in each culture, $30 \mu \mathrm{g} . / \mathrm{ml}$. medium.

$37^{\circ}$ with aeration. Samples were removed at intervals, optical densities recorded and radioactivity assays made of the washed organisms. At all times the uptake of isotope by the control culture (containing ${ }^{32} \mathrm{~S}$-sulphate) was directly proportional to the amount of growth as measured by the optical density. Table 3 shows the relative radioactive uptakes of cultures containing other sulphur sources after $2.5 \mathrm{hr}$. growth, and indicates that cysteine, 
thiosulphate and dithionite were preferred sulphur sources, in that sulphate uptake was decreased in the presence of equimolar concentrations of these compounds. Methionine or sulphite decreased the uptake to a lesser extent, and cysteic acid had little effect. The results of similar experiments using sulphate at $30 \mu \mathrm{g} . / \mathrm{ml}$. and $0.5 \mu \mathrm{c} / \mathrm{ml}$. and certain competitors at $3 \mu \mathrm{g} . / \mathrm{ml}$. are also shown in Table 3.

\section{Biochemical heterogeneity within cysB group}

From Table 2 it will be seen that the group of mutants designated cys $B$ can be defined nutritionally by their inability to give detectable growth on low $(10 \mu \mathrm{g}$. $\mathrm{S} / \mathrm{ml}$.) concentrations of either sulphite or thiosulphate, although they utilize thiosulphate at higher ( $1 \mathrm{mg}$. S/ml.) concentrations after incubation for $18 \mathrm{hr}$.

(i)

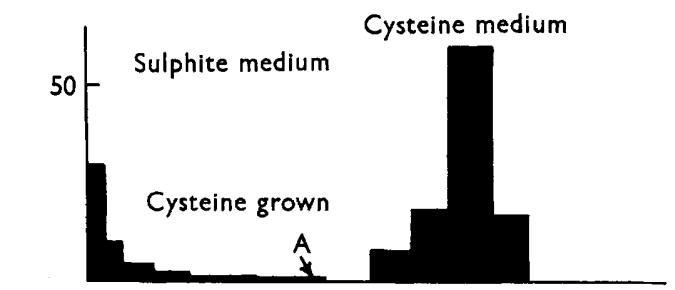

(ii)

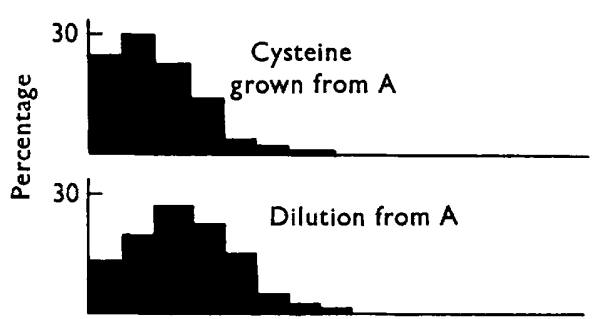

(iv)

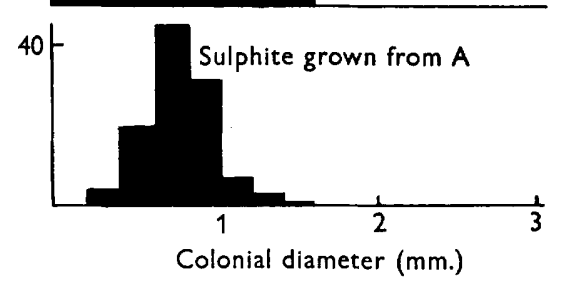

Fig. 3. Distribution of colony sizes obtained from a culture of $c y s B-18$ plated on minimal medium containing sulphite after the following treatments:

(i) Growth in cysteine-supplemented minimal medium (cysteine grown). Selection of a colony (A) after above treatment having a maximal size on sulphite medium (1$1.25 \mathrm{~mm}$.) and replating on minimal sulphite medium after:

(ii) Growth in cysteine-supplemented liquid medium (cysteine grown from A).

(iii) Suspension in saline (dilution from 'A').

(iv) Growth on sulphite-supplemented liquid medium (sulphite grown from A).

Growth on cysteine-supplemented minimal medium was similar after all treatments and is illustrated in top right histogram.

However, when the duration of incubation on sulphite- or thiosulphatecontaining $(10 \mu \mathrm{g}$. $/ \mathrm{ml}$.) medium was increased to $40 \mathrm{hr}$., certain of the mutants (cysB-1 0,-18, -24 and -25) when spread at high densities, gave isolated colonies, 
whereas no other mutants of this group gave any perceptible growth. Since there appeared to be no concomitant growth on sulphate to suggest 'leakiness', the phenomenon was further investigated as a result of which the cys $B$ mutants are subdivided into three secondary phenotypes.

First phenotype. A single clone of $c y s B-18$ was grown to saturation in cysteine-supplemented medium, and after centrifugation and dilution, plated on sulphite-containing minimal medium. (In all experiments, the diluted samples plated on sulphite medium were also plated on cysteine medium to give a count of viable organisms, and on sulphate medium to give a control of reversion or leakiness.) After incubation for $48 \mathrm{hr}$. at $37^{\circ}$ c. $0.5 \%$ of the organisms plated gave colonies with a diameter of about half $(1.5 \mathrm{~mm}$.) that formed on cysteine $(3 \mathrm{~mm}$.$) . Another 0.5 \%$ of the organisms produced colonies which were $\mathbf{0} \cdot \mathbf{8}-\mathbf{1} \cdot \mathbf{5} \mathrm{mm}$. in diameter, and a further $\mathbf{5 0} \%$ gave colonies of diameters ranging from 50 to $800 \mu$. The total colonies, representing about $50 \%$ of the number of colonies on parallel platings on cysteine medium, appeared to show a continuous variation in size from a minimum of $50 \mu$. to a maximum of $1.5 \mathrm{~mm}$. On further incubation up to 5 days the colonial sizes increased proportionally to a maximum diameter of $3 \mathrm{~mm}$., but there was little increase in the total number of colonies, and the continuous variation in size was maintained. Several of the large colonies were subcultured on sulphite medium, either immediately after dilution in saline, or after an intermediate period of growth for $18 \mathrm{hr}$. at $37^{\circ}$ from a small inoculum $(c .200$ organisms $/ \mathrm{ml}$.) in either cysteine or sulphite-supplemented liquid minimal medium. The distributions of colonial sizes on sulphite-containing medium after these treatments are summarized in Fig. 3. In no cases were colonies produced on sulphate-supplemented medium. In a limited number of experiments, mutants cys-24 and -25 showed characteristics similar to those of cys-18 described above, as did all three strains on thiosulphate medium. No increase in growth was detected when any of these strains was aerated for periods up to $18 \mathrm{hr}$. at $37^{\circ}$ in liquid minimal media + sulphite or thiosulphate.

Second phenotype. This was exhibited by cys $B-10$. Cultures grown in cysteine liquid minimal medium gave colonies of uniform size when plated on either sulphite- or thiosulphate-supplemented minimal media, although the colony diameter was only a quarter of that resulting after growth under the same conditions on cysteine medium. The number of colonies was similar on all three media. Growth curves in sulphite-supplemented medium showed no increase in lag and a slow steady growth with a mean generation time $c .10 \mathrm{hr}$.

Third phenotype. This is as originally described: there is no perceptible growth on sulphite or thiosulphate, except when the concentration of the latter salt is raised to $1 \mathrm{mg} . \mathrm{S} / \mathrm{ml}$. This phenotype is characteristic of the remaining members of the cysB group (cysB-4,-12,-14,-15,-16,-27, $-40,-41$ and -45$)$.

When cys $C$ mutants were plated on the sulphite- or thiosulphate-supplemented media used in these experiments, colonies of the same number and size as those found on cysteine-supplemented medium resulted. 


\section{DISCUSSION}

From the present studies the existence of a class of mutants (cys $A$ ) which use sulphite equiv. $10 \mu \mathrm{g} . \mathrm{S} / \mathrm{ml}$. but not thiosulphate (except at a hundred-fold concentration) makes it seem unlikely that in Salmonella, cyst(e)ine is formed from sulphate through an unbranched reductive pathway. Hockenhull (1949) suggested that sulphite may be reduced in one of several ways. The simplest route is its direct reduction through the metabisulphite $\left(\mathrm{S}_{2} \mathrm{O}_{5}{ }^{\prime \prime}\right)$ and dithionite $\left(\mathrm{S}_{2} \mathrm{O}_{4}{ }^{\prime \prime}\right)$ to thiosulphate, but it may alternatively be reduced to the unstable sulphoxylate $\left(\mathrm{SO}_{2}{ }^{\prime \prime}\right)$. This latter compound may then either dimerize to form thiosulphate, or react non-enzymically with further sulphite to give dithionite, which is then spontaneously reduced by more sulphoxylate to thiosulphate.

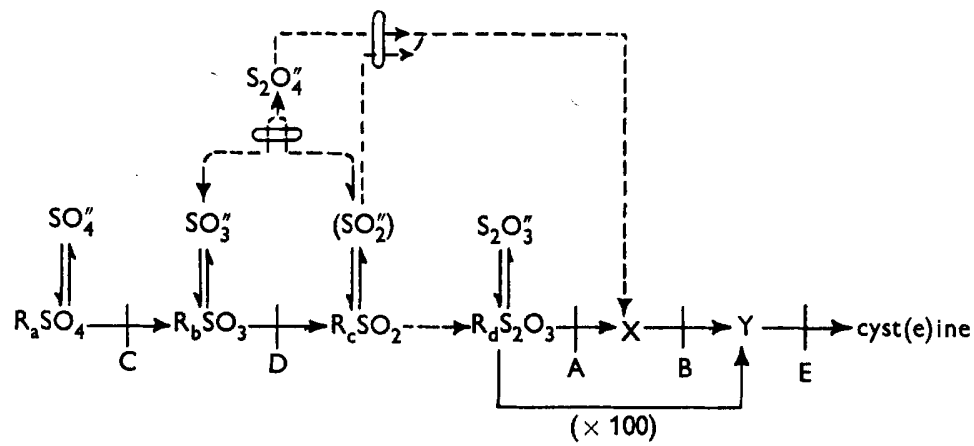

Fig. 4. Schematic diagram to represent possible pathway of sulphur metabolism in Salmonella typhimurium. Interrupted lines represent reactions which may occur in the absence of enzymes. $\mathbf{R}_{a}, \mathbf{R}_{b}$, etc., represent binding sites or non-diffusible organic radicals holding diffusible ions within organisms. $A, B, C, D$ and $E$ represent the location of metabolic blocks. Flattened circles denote that the participation of the two reactants is necessary for the reaction to proceed in the direction of the arrows.

Thus sulphoxylate, once formed, may be converted to thiosulphate in one of two ways without enzyme participation or further reduction. These interconversions, included in Fig. 4, appear to offer a satisfactory basis for summarizing the growth responses and other data obtained with the cyst(e)ineless Salmonella mutants if one assumption be made, namely, that dithionite may be reduced by sulphoxylate to give, not thiosulphate, but rather a subsequent intermediate denoted as ' $X$ '. In this compound the sulphur atom would not have already been incorporated into the cyst(e)ine carbon chain, and since one of the sulphur atoms of the thiosulphate molecule is in the lowest reduction state, the conversion of thiosulphate to ' $\mathrm{X}$ ' need not necessarily be by reduction. The interrupted lines in Fig. 4 denote those reactions which, according to the evidence summarized by Hockenhull, may proceed in the absence of enzymes, and which would correspond to those steps which do not appear to be under genetic control, since no mutants have been isolated with a phenotype indicating a metabolic block at these points.

According to this scheme (Fig. 4), cysC mutants, blocked between sulphate and sulphite, would be expected to grow at a rate equivalent to wild-type on 
either sulphite or thiosulphate, by utilizing either pathway. $C y s D$ mutants could also utilize thiosulphate by the direct pathway, but could not utilize sulphite, being unable to convert this compound to sulphoxylate. On dithionite, cys $D$ mutants would be required to make use of the shunt pathway. Cys $A$ mutants could not utilize thiosulphate, but could utilize dithionite via the auxiliary pathway, and sulphite also by this pathway, since reduction of sulphite to sulphoxylate is possible, because of the integrity in $c y s A$ mutants of the step which is shown blocked at ' $\mathrm{D}$ ' in $c y s D$ mutants (Fig. 4). CysB and cys $E$ mutants would not be expected to utilize any of these inorganic salts as a source of sulphur. These metabolic potentialities are equivalent to those found by experiment and summarized in Figs. 1 and 2, and constitute the main evidence in favour of the scheme represented in Fig. 4.

The ability of $c y s C$ mutants to grow at the wild-type rate on dithionite may be due to its conversion to sulphite and sulphoxylate, which, according to Newton-Friend (1947), proceeds at slightly acid $\mathrm{pH}$ values. That $c y s D$ mutants do not appear to be able to utilize this pathway of dithionite metabolism may be due to the accumulation of sulphite which cannot be further reduced in this direction.

Syntrophism by certain $c y s D$ mutants $\left(D_{1}\right)$ mutants could presumably be due to the accumulation of sulphite-containing intermediates which can be utilized by either cysC or cys $A$ mutants, and similarly the ability of cys $A$ mutants to feed cysC mutants might well be due to the accumulation of thiosulphate-like intermediates. This accumulation should also support the growth of cysD mutants, but this is unable to be demonstrated in the presence of non-specific syntrophism of these mutants with the wild-type strain and all other mutants. The inability of the other cys $D$ mutants $\left(D_{2}\right.$ mutants) to support growth of either cysC or cys $A$ mutants is not readily explained from the biochemical data.

In addition, there appears to be a by-pass reaction not normally operative, but one in which the thiosulphate may be converted to the unknown intermediate ' $\mathrm{Y}$ ' further along the pathway, allowing $c y s \boldsymbol{A}$ and $c y s B$ mutants to utilize thiosulphate when this is supplied at a concentration a hundred times greater than that normally utilizable. Certain mutants of Aspergillus nidulans have also been found, by Hockenhull (personal communication) to require concentrations of thiosulphate of 50-100 $\mathrm{g}$. S $/ \mathrm{ml}$. for growth.

An alternative explanation for the inability of $c y s A$ mutants to be accommodated within a simple unbranched pathway of sulphate reduction through sulphite and thiosulphate respectively, by reason of their inability to utilize thiosulphate (at $10 \mu \mathrm{g} . / \mathrm{ml}$.) might be supposed to be an impermeability of these mutants to thiosulphate, since growth can be demonstrated at the $1 \mathrm{mg} . /$ ml. level. However, this would imply that the purported single mutational event was capable of producing impermeability to thiosulphate, together with either an impermeability to sulphate or a metabolic block between sulphate and cyst(e)ine, since there is no growth on sulphate. The immediate uptake of radiosulphate by wild-type and mutant in the absence of growth, measured by the technique of Roberts et al. (1955) corresponded to between 65 and $75 \%$ 
of the volume of the organism, and no difference in this uptake could be detected between $c y s A$ mutants and those of other mutants or of wild-type Salmonella, suggesting that there is little difference in the permeability of any of these strains to sulphate. An unbranched sequence of sulphate reduction would therefore require that all cys $A$ mutants are impermeable to thiosulphate, and in addition have a metabolic block in the pathway of reduction of sulphate to cyst(e)ine.

Previously reported experiments with cysteine sulphinic acid support the concept of an inorganic pathway at least as far as thiosulphate in all organisms except Aspergillus nidulans (Shepherd, 1956). In this latter study, alternative inorganic and organic pathways of sulphur reduction were suggested, but the proposed scheme required one of the mutants to have simultaneous metabolic blocks in two of the pathways concerned. Moreover, in Escherichia coli the ability of pyruvate to supply the carbon source for cyst(e)ine synthesis, as postulated by Shepherd, was considered unlikely by Roberts et al. (1955) because of its inability to compete with glucose for this function, whereas under the same conditions serine was able almost entirely to suppress the contribution of carbon from glucose to cyst(e)ine.

The concept of an inorganic pathway of reduction is supported by the present studies, since no accumulation of either cysteic acid or cysteine sulphinic acid was detected in the supernatant fluid or disrupted cells of representative mutants or wild-type grown in the presence of radiosulphate and tested chromatographically by the methods of Chapeville \& Fromageot (1954), even though these disrupted cell preparations were active in stimulating the growth of other mutants as detailed previously. The inability of sulphide and free sulphur to give adequate growth responses supports similar findings by Hockenhull and by Steinberg, who suggested that these compounds were unlikely to be direct intermediates. The isotopic competition experiments suggest that thiosulphate in particular is a direct intermediate in view of its ability almost completely to exclude sulphate uptake on an equimolar basis. The ability of this compound to act as a competitor to sulphate was even greater in the experiments of Roberts et al. (1955) with Escherichia coli. However, it would appear that the demonstration by these same authors of the ability of sulphite to suppress sulphate uptake to a much greater extent in $\boldsymbol{E}$. coli than was found in Salmonella suggests a different mechanism of participation of sulphite in the two systems. Moreover, Salmonella typhimurium appears to differ from previously studied organisms by its inability to utilize cysteic acid as a sulphur source.

The lack of apparent syntrophism except after 3 days of incubation at $37^{\circ}$ with growing organisms and the presence of growth-promoting substances within the cells even after a dilution of $1 / 500$, compared with the inactivity of the corresponding supernatant fluids suggests that these intermediates are not diffusible, and are normally liberated only after autolysis. It would appear, therefore, that if they are inorganic ions they do not exist in a free state but may be bound by some large non-diffusible organic radicals, at sites within the cell (Roberts et al. 1955), which are denoted as $R_{a}, R_{b}$ etc. in Fig. 4. 
The mutants cys-10, $-18,-24$ and -25 , classified provisionally as $c y s B$ mutants on the basis of primary nutrition in Table 2 , appear on further investigation to be intermediate in phenotype to $c y s B$ and $c y s C$, and cannot be easily accommodated within the proposed pathway.

My thanks are due to Dr M. Demerec, for providing the oyst(e)ineless mutants, and for facilities to work during the tenure of a Damon Runyon Cancer Research Fellowship; to the members of the Department of Terrestrial Magnetism, Carnegie Institution of Washington, for help and advice with the radioactive studies, and to the Wright-Fleming Institute of Microbiology, St Mary's Hospital, London, for leave of absence.

\section{REFERENCES}

Chapeville, F. \& Fromageot, P. (1954). La formation enzymatique de l'acide cystinesulfinique à partir de sulfite. Biochim. biophys. Acta, 14, 415 .

Demerec, M. (1956). Terminology and Nomenclature. In Genetic Experiments with Bacteria. Publ. Carneg. Inst. 612, 1.

Demerec, M. \& CahN, E. (1953). Studies on mutability in nutritionally deficient strains of Escherichia coli. 1. Genetic analysis of five auxotrophic strains. J. Bact. 65, 27.

Hershey, A. D. (1955). An upper limit to the protein content of the germinal substance of bacteriophage T2. Virology, 1, 108.

Hockenhuld, D. J. D. (1949). The sulfur metabolism of mold fungi. The use of biochemical mutant strains of Aspergillus nidulans in elucidating the biosynthesis of cystine. Biochim. biophys. Acta, 3, 326.

Horowitz, N. H. (1950). Biochemical genetics of Neurospora. Advanc. Genet. 3, 33.

Hовоwiтz, N. H. (1955). Symposium on Amino acid Metabolism. Discussion, 631. Baltimore: The Johns Hopkins Press.

Lampen, J. O., Roepke, R. R. \& Jones, M. J. (1947). Studies on the sulfur metabolism of E. coli. III. Mutant strains of $E$. coli unable to use sulfate for their complete sulfur requirements. Arch. Biochem. 13, 55.

LEDERBERG, J. (1950). Isolation and characterization of biochemical mutants of bacteria. Meth. med. Res. 3, 5.

Newton Friend, J. (ed.) (1947). Textbook of Inorganic Chemistry, 7, 124. London: Griffin.

Phinney, B. O. (1948). Abstract in Genetics, 33, 624.

Roberts, R. B., Abelson, P. H., Cowie, D. B., Bolton, E. T. \& Britten, R. J. (1955). Studies of biosynthesis in Escherichia coli. Publ. Carneg. Inst. 607, Washington, D.C.

Shepherd, C. J. (1956). Pathways of cysteine synthesis in Aspergillus nidulans. J. gen. Microbiol. 15, 29.

Simmonds, S. (1948). Utilization of sulfur containing amino acids by mutant strains of Escherichia coli. J. biol. Chem. 174, 717.

Steinberg, R. A. (1941). Sulfur and trace element nutrition of Aspergillus niger. J. Agric. Res. 63, 109.

Zinder, N. D. \& Lederberg, J. (1952). Genetic exchange in Salmonella. J. Bact. 64, 679 . 